\title{
Saccade disturbances after bilateral lentiform nucleus lesions in humans
}

\author{
A-I Vermersch, R M Müri, S Rivaud, $M$ Vidailhet, B Gaymard, Y Agid, \\ C Pierrot-Deseilligny
}

\begin{abstract}
Objective-To determine the roles of the putamen and pallidum in ocular motor control.

Methods-Eye movements were recorded electro-oculographically in nine patients with bilateral focal lesions affecting the lentiform nucleus, and in 12 age matched control subjects. Reflexive visually guided saccades (gap task), antisaccades, memorised sequences of saccades, memory guided saccades (with visual input only, and with both visual and vestibular inputs), and predictive saccades (with and without gap) were studied.

Results-Latency and accuracy of visually guided saccades were normal. The percentage of errors in the antisaccade task and latency of correct antisaccades did not differ significantly from the results of controls. The percentage of errors in saccade sequences was significantly increased. Accuracy of the two types of memory guided saccades was impaired bilaterally. The percentage of predictive saccades was significantly decreased when a gap existed, but unchanged without a gap, compared with controls. Therefore, saccades made immediately in response to an external target (reflexive visually guided saccades and antisaccades) were performed without difficulty, whereas those requiring an internal representation of such a target (such as memory guided saccades, predictive saccades, and saccade sequences) were performed with significant disturbances.
\end{abstract}

Conclusions-The lentiform nucleus influences the cortical areas involved in the control of saccades when the experimental paradigm requires the use of an internal representation of the target for correct planning and execution of the ensuing saccade.

$(\mathcal{F}$ Neurol Neurosurg Psychiatry 1996;60:179-184)

Keywords: saccades; putamen; pallidum

In the frontal lobe, there are three cortical areas involved in the control of saccades ${ }^{1}$ : the frontal eye field, the supplementary eye field, and the dorsolateral prefrontal cortex. The basal ganglia are connected to cortical areas through different neuronal circuits. ${ }^{2}$ The frontal cortical areas receive direct feedbacks from the basal ganglia, in particular through a pathway involving successively the putamen, the pallidum, and the thalamus. ${ }^{3-5}$ There is evidence from electrophysiological studies that the motor circuit involving the basal ganglia and thalamus has an important role in modulating the planning and execution of limb movements. ${ }^{6}$ In humans, bilateral focal lesions of the lentiform nucleus - that is, affecting the putamen and/or the pallidum, cause motor disorders such as dystonia and, less often, behavioural disturbances such as abulia or disinhibition. ${ }^{7}$ Positron emission tomography in such patients shows hypometabolism in the prefrontal cortex, suggesting a deafferentation of this cortex resulting from damage to the lentiform nucleus.

The precise role of the lentiform nuclei in the control of eye movements is still unclear. In monkeys, there are some anatomical data concerning the ocular motor corticosubcortical pathways ${ }^{6}$ but no studies of the consequences of lesions in the lentiform nucleus for eye movements. In humans, bilateral focal lesions affecting this nucleus are $\operatorname{rare}^{7}$ and, to our knowledge, there have been no previous reports of recordings of eye movement in patients with such lesions. To determine the role of the putamen and pallidum in ocular motor control, we studied nine patients with bilateral lesions of the lentiform nuclei, using seven saccade tasks: reflexive visually guided saccades, antisaccades, two types of memory guided saccades, saccade sequences, and two types of predictive saccades.

\section{Methods}

SUBJECTS

Nine patients (five men and four women; age: 44 (SD 11) years) with bilateral lesions in the lentiform nucleus were examined. The lesions, documented by MRI in eight patients and CT in one patient, were clearly visible and apparently limited to the lentiform nucleus, without visible damage to the internal capsule or other cerebral structures (fig 1). The aetiology of these lesions was cardiac arrest $(n=2)$, anoxia $(\mathrm{n}=3)$, and carbon monoxide intoxication $(n=2)$. The mean delay between the cause of brain damage and the eye movement recording was 13 (range 1 to 44 ) years in these seven patients. In the remaining two patients, the aetiology and age of the lesions were unknown. In four patients, the bilateral lesions concerned predominantly the pallidum and in five patients predominantly the putamen (fig 2). Clinically, all patients with lesions affecting predominantly the putamen and two patients 
Figure 1 Horizontal MRI section of patient 1 showing hypersignals in both putamen areas.

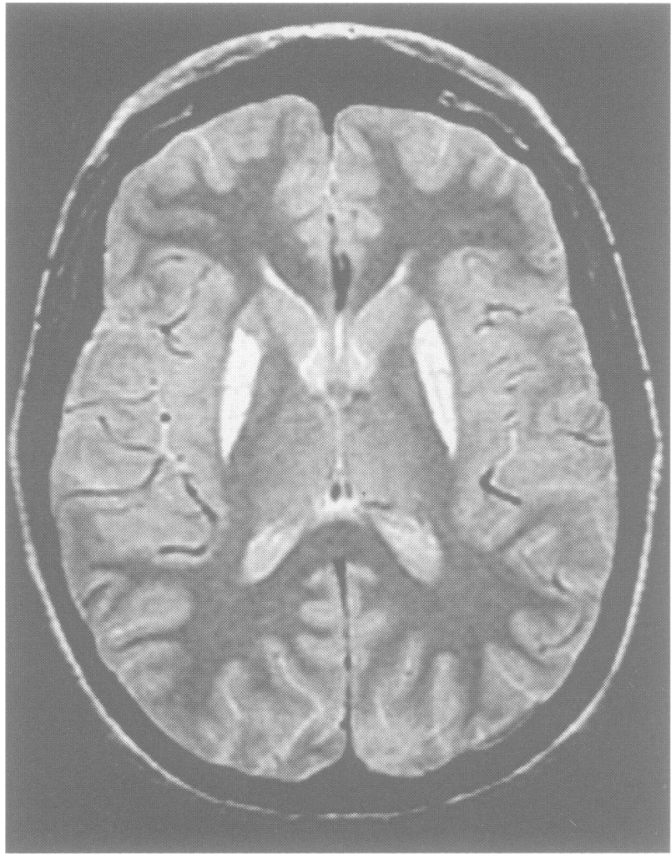

with lesions affecting both the putamen and pallidum had focal or generalised dystonia. Two of the four patients with predominantly pallidal lesions showed no neurological signs, the third patient presented with a writing tremor, and the fourth showed behavioural disturbances such as inertia and loss of drive. None of the nine patients had kinetic or static cerebellar syndromes, intellectual deterioration, episodic memory deficit ${ }^{9}$ or depression.
An age matched control group of 12 normal subjects (seven men and five women; mean age 47 (SD 10) years) was also studied, with the same paradigms as in the patient group. Informed consent was obtained from all patients and control subjects.

\section{PROCEDURE}

Eye movements were recorded by direct current electro-oculography, in complete darkness with bitemporal electrodes. The bandwidth of the recording amplifiers was from 0 to $100 \mathrm{~Hz}$ and the system had a resolution of $1^{\circ}$. The subject's head was immobilised. Stimulation was carried out using a curvilinear ramp of light emitting diodes (LEDs) located $80 \mathrm{~cm}$ in front of the subject. Horizontal saccades were studied in seven different tasks, as described below.

Visually guided saccades (fig $3 A$ )

In this task, the central fixation point was switched off $200 \mathrm{~ms}$ (gap) before the onset of a luminous lateral target located $25^{\circ}$ to right or left of the central point. The subject was instructed to fixate the central point and to look at the lateral target as soon as it appeared. The target was presented pseudorandomly right or left, with unpredictable timing. Left and right saccade latencies were calculated for each subject by averaging 20 measurements in each direction. Saccade accuracy was determined using the primary saccade and the percentage of error in amplitude of this saccade (target eccentricity minus saccade amplitude divided by target eccentricity).
Figure 2 Schematic representation of the lentiform nucleus lesions in the nine patients studied. The hatched areas represent the lentiform nuclei, and the lesions are shown in black. Pal $=$ pallidum; Put $=$ putamen.
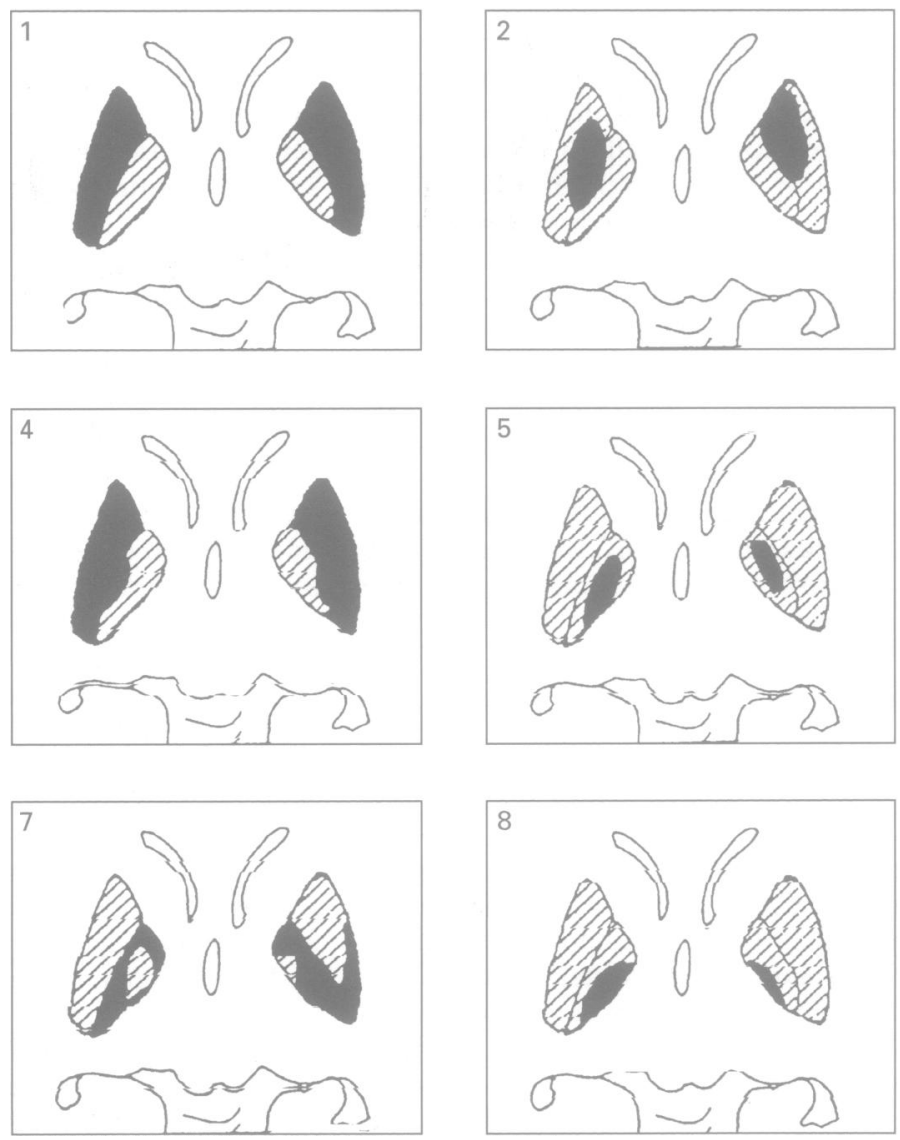

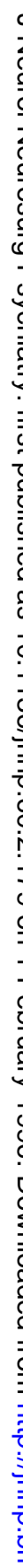

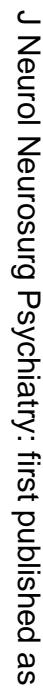

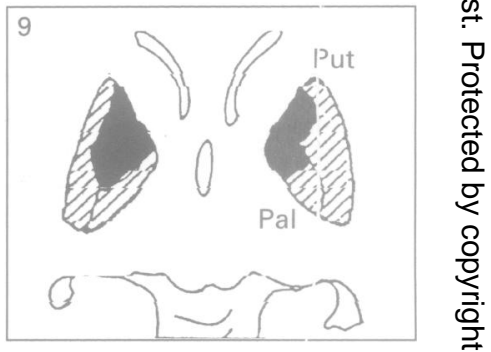


Figure 3 Saccade paradigms (A) Visually guided saccades with a gap: saccade latency (Lat) and amplitude (am) were

measured. (B)

Antisaccades: latency of correct antisaccades (Lat) and the percentage of errors (e) (misdirected saccades) were determined. $(C)$ Memorised sequences of saccades $(1,2,3,4=$ sequence of targets): the percentage of incorrect sequences (errors in the order or number of saccades) was calculated. Note that the saccade sequence shown is incorrect (inversion of the third and fourth saccades). (D)

Memory guided saccades,

(a) with visual input and

(b) with visual and

vestibular input: amplitude (am) was measured ( $c=$ corrective saccade). (E)

Predictive saccades, (a) with gap and (b) without gap: the percentage of predictive saccades (arrows) was determined. $F=$ central fixation point; $G=$ gap $; H=$ head $L=$ left; $M=$ midline; $R=$ right $; T=$ target.
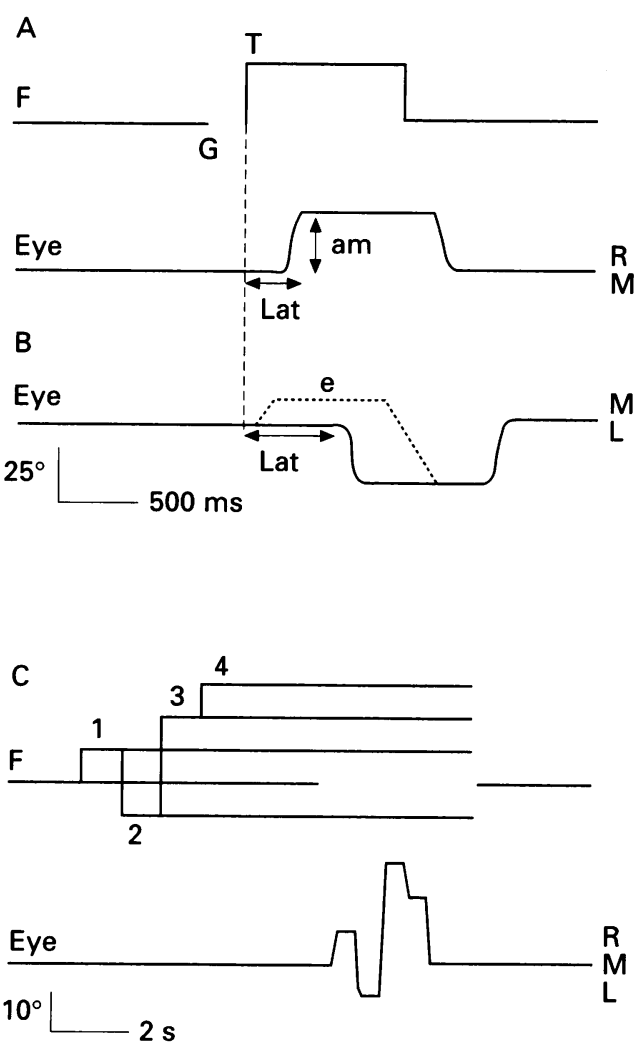

Antisaccades (fig 3B)

The procedure was the same as in the gap task except that the subject was instructed to look in the opposite direction to the suddenly appearing lateral target, without first looking at the target. Twenty trials were made in each lateral direction. Latency of correct antisaccades and the percentage of errors (misdirected saccades-that is, reaching or simply initially directed towards the target) were determined for each lateral direction.

\section{Memorised sequences of saccades (fig 3C)}

The subject fixed a central fixation point while four lateral LEDs were switched on, successively at one second intervals, and remained illuminated. The eccentricity of the targets was between $8^{\circ}$ and $32^{\circ}$ from the midline. The subject was instructed to remember the order of appearance of the LEDs without looking at them. Three seconds after the last LED had been switched on, the central fixation point was switched off and the subject then had to look successively at the four LEDs in the same order as stimulation and with about the same cadence (with a pause of about one second at each target location). For both patients and controls, a few sequences were used to explain the task. A sequence was considered incorrect if there was at least one error in the order or number of executed saccades. Twenty different sequences were performed and the percentage of incorrect sequences was calculated.

Memory guided saccades (fig 3D)

Two different types of memory guided saccades were studied. In both cases, the subject was instructed to look at a central fixation point while a single lateral target was flashed

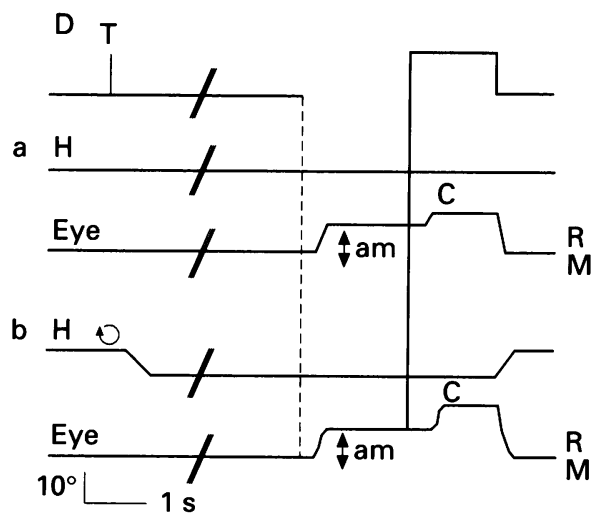

E
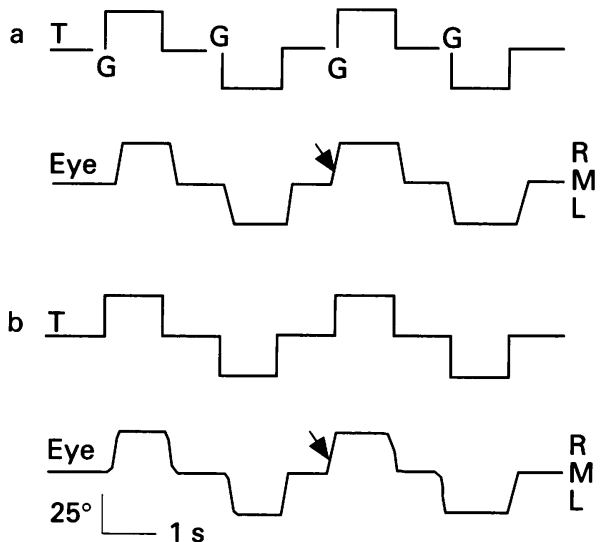

for $50 \mathrm{~ms}$, to right or left, with unpredictable timing and eccentricity $\left(10^{\circ}, 15^{\circ}, 20^{\circ}, 25^{\circ}\right.$, or $30^{\circ}$ ). The subject had to continue to look at the central fixation point during a six second delay. After this delay, the central fixation point was switched off, and the subject made a saccade, in complete darkness, to the remembered location of the flash. Then, a lateral target with the same position as the flash was switched on and a corrective saccade was made, if necessary. In the first task, the central fixation point remained immobile and the memory guided saccade was made with only a visual input. In the second task, the subject was rotated during the six second delay (the head being fixed with respect to the trunk), while continuing to look at the central fixation point rotating with the chair. This resulted in a suppression of the vestibulo-ocular reflex. In this case, the memory guided saccade had both a visual and vestibular input. The direction and amplitude of rotation was unpredictable (between $5^{\circ}$ and $15^{\circ}$ ); however, chair rotation never reached or exceeded the eccentricity of the flash. In both tasks, saccade accuracy was determined using the primary saccade and the percentage of error in amplitude of this saccade, which was calculated by averaging 15 measurements in each direction.

Predictive saccades (fig $3 E$ )

Two different types of predictive saccades were studied. In each case, the subject was instructed to follow luminous targets which were presented successively, at one second intervals $25^{\circ}$ leftwards, in the centre, $25^{\circ}$ rightwards, and again the centre. Eighteen cycles were performed without interruption. In the first predictive saccade task a 200 ms gap was 
introduced between targets for the stimulation of centrifugal saccades (predictive saccades with gap). In the second task, there was no gap (predictive saccades without gap). Thus target direction, amplitude, and timing were predictable in both tasks. A saccade was considered to be predictive and not visually guided when latency was less than $70 \mathrm{~ms} .{ }^{10} \mathrm{In}$ both tasks, the percentage of centrifugal predictive saccades was calculated in each direction.

Statistical analysis was performed with the Mann-Whitney $U$ test to compare the results of the patient group with those of the control group.

\section{Results}

In a first analysis (not reported), the patient group was divided into two subgroups (patients with predominantly putamen lesions and patients with predominantly pallidum lesions) and the results of these two subgroups were compared. Because results of these two subgroups were analogous (with no significant differences), all nine patients were thereafter studied as a single group, and their results compared with those of the control group (table). In the patient group, latency of visually guided saccades was on average 169 ms rightwards and $178 \mathrm{~ms}$ leftwards, which was not significantly different from latency in the control group (198 ms for rightward saccades and $186 \mathrm{~ms}$ for leftward saccades). Furthermore, there was no significant difference in the percentage of error in amplitude ( $9 \%$ on average in patients $v 5 \%$ in controls for rightward saccades, and $10 \% v 7 \%$ in controls for leftward saccades).

In the patient group, latency of antisaccades was on average $300 \mathrm{~ms}$ rightwards and $327 \mathrm{~ms}$ leftwards, which was not significantly different from mean latency in the control group (262 $\mathrm{ms}$ for rightward saccades and $253 \mathrm{~ms}$ for leftward saccades). The percentage of misdirected saccades in the antisaccade task was on average $18 \%$ rightwards, and $13 \%$ leftwards - that is, not significantly different from that of controls ( $9 \%$ and $13 \%$, respectively).

The percentage of incorrect sequences of memory guided saccades was significantly increased $(P<0.001)$ in patients (mean 54\%), compared with that of controls $(23 \%)$.

The percentage of error in amplitude of memory guided saccades with visual input was significantly increased: $27 \%$ on average for rightward and $31 \%$ for leftward saccades (controls: $18 \%$ for rightward and $16 \%$ for leftward saccades, $\mathrm{P}<0.02$ and $\mathrm{P}<0.002$ respectively). Significant differences were also found for memory guided saccades with visual and vestibular input: $40 \%$ on average for rightward saccades and $40 \%$ for leftward saccades (controls: $20 \%$ for rightward and $23 \%$ for leftward saccades, with $P<0.004$ and $P<0.003$ respectively).

In the control group, the percentage of predictive saccades was increased in the task with a gap, compared with that of the task without a gap: $88 \%$ on average for rightward saccades with a gap $v 53 \%$ without a gap $(\mathrm{P}<0.004)$, and $93 \%$ for leftward saccades with a gap $v$ $46 \%$ without a gap $(P<0.001)$. In the patient group, the percentage of predictive saccades without a gap did not differ significantly from that of controls (43\% on average for rightward saccades $v 53 \%$ in controls, and $42 \%$ for leftward saccades $v 46 \%$ in controls). By contrast, the percentage of predictive saccades in the task with a gap was significantly lower than that controls: $57 \%$ on average for rightward saccades $v 88 \%$ in controls $(\mathrm{P}<0.01)$ and $61 \%$ for leftward saccades $v 93 \%$ in controls $(P<0.02)$. Therefore, by contrast with the results of the control group, the percentage of predictive saccades in the patient group did not increase significantly in the task with a gap compared with that in the task without a gap.

\section{Discussion}

In our patients, the lesions visible on MRI were probably necrotic, given their aetiology, and limited to the lentiform nuclei, which are

Comparison of horizontal saccade tasks between patients and controls

\begin{tabular}{|c|c|c|c|c|}
\hline & Side & $\begin{array}{l}\text { Controls } \\
\text { Mean (SD) or (range) }\end{array}$ & $\begin{array}{l}\text { Patients } \\
\text { Mean (SD) or (range) }\end{array}$ & Pvalue \\
\hline \multicolumn{5}{|l|}{ Visually guided saccades: } \\
\hline Latency (ms) & Right & $\begin{array}{l}198(35) \\
186(53)\end{array}$ & $169(28)$ & NS \\
\hline \multirow{2}{*}{ Accuracy (percentage of error in amplitude) } & Right & $5(3)$ & $9(4)$ & NS \\
\hline & Left & $7(6)$ & $10(5)$ & NS \\
\hline \multicolumn{5}{|l|}{ Antisaccades: } \\
\hline Latency of correct saccades (ms) & Right & $262(42)$ & $327(84)$ & NS \\
\hline \multirow[t]{2}{*}{$\%$ of misdirected saccades } & Right & $9(0-30)$ & $18(0-33)$ & NS \\
\hline & Left & $13(0-33)$ & $13(0-29)$ & NS \\
\hline \multirow{2}{*}{$\begin{array}{l}\text { Sequences of memory guided saccades } \\
\% \text { of errors } \\
\text { Memory guided saccades: }\end{array}$} & & $23(10-35)$ & $54(44-80)$ & $<0.001$ \\
\hline & & & & \\
\hline \multicolumn{5}{|l|}{ Visual input } \\
\hline Accuracy (percentage of error in amplitude) & $\begin{array}{l}\text { Right } \\
\text { Left }\end{array}$ & $\begin{array}{l}18(7) \\
16(7)\end{array}$ & $\begin{array}{l}27(11) \\
31(10)\end{array}$ & $\begin{array}{l}<0.02 \\
<0.002\end{array}$ \\
\hline \multirow{2}{*}{$\begin{array}{l}\text { Visual and vestibular input } \\
\text { Accuracy (percentage of error in amplitude) }\end{array}$} & & & & \\
\hline & Right & $20(8)$ & $40(15)$ & $<0.004$ \\
\hline & Left & $23(10)$ & $40(4)$ & $<0.003$ \\
\hline \multicolumn{5}{|l|}{ Predictive saccades: } \\
\hline \multirow[t]{2}{*}{$\%$ of predictive saccades } & Right & $88(47-100)$ & $57(24-82)$ & $<0.018$ \\
\hline & Left & $93(65-100)$ & $61(0-88)$ & $<0.014$ \\
\hline \multirow{2}{*}{$\begin{array}{l}\text { Without gap } \\
\% \text { of predictive saccades }\end{array}$} & Right & $53(6-100)$ & $43(0-89)$ & \\
\hline & Left & $46(6-100)$ & $42(9-19)$ & NS \\
\hline
\end{tabular}


known to be particularly sensitive to anoxia. Although cerebral damage may initially have been more extensive, it can be hypothesised for several reasons that the residual lesions affected principally the lentiform nuclei. (1) There were no other visible lesions on MRI. (2) Damage to the lentiform nuclei alone would be sufficient to account for all the associated clinical signs (dystonia, inertia and loss of drive $)^{78}$; in particular, there was no clinical evidence of cerebellar or memory impairmentthat is, of functional damage to the cerebellum and hippocampal region, two structures also known to be sensitive to anoxia and involved in eye movement control. (3) The mean delay between the onset of brain damage and eye movement recordings was long (13 years); this suggests that the effect of any slight damage, which may have initially existed in addition to the lentiform nucleus lesions, could have been only transitory and have disappeared long before our study or could have largely improved by different compensation mechanisms after such a period. (4) In support of limited subcortical lesions in our patients, it should be noted that, in a clinicopathological study of 22 patients with carbon monoxide intoxication, there was no cortical damage in most cases $^{11}$; furthermore, anatomical details of two similar patients showed that the cytoarchitecture of the frontal cortex was preserved. ${ }^{12}$ Therefore, it may be assumed that the ocular motor disturbances in our patients were, largely if not entirely, related to the bilateral lentiform nucleus lesions visible on MRI.

There were no obvious differences in the performance of saccade tasks between patients with damage predominantly to the pallidum and those with damage affecting preferentially the putamen. It may well be that lesions restricted to the putamen or the pallidum do not exist in such a pathology. Furthermore, the putamen and pallidum may belong to the same neuronal circuit projecting (via the thalamus) to frontal areas involved in the control of saccades. ${ }^{6}$ Therefore, damage to either structure could result in similar ocular motor disturbances.

The seven different saccade paradigms studied here can be divided into two groups: (1) saccades performed immediately (reflexive visually guided saccades and antisaccades), without the need for additional, internal information; and (2) saccades which require the use of internal, memorised spatial or temporal information (memory guided and predictive saccades, and sequences of memory guided saccades). In patients with bilateral lesions of the lentiform nucleus, the first group of tasks was performed without difficulty, whereas the performance of the second group of tasks was impaired.

The posterior eye field and the frontal eye field are involved in the cortical control of reflexive visually guided saccades. ${ }^{13} 14$ The preservation of such saccades suggests that they are not controlled by the putamen or pallidum. These saccades could be controlled by direct corticocollicular pathways, ${ }^{1516}$ and an indirect, corticocaudo-nigrocollicular path- way. ${ }^{217}$ In the antisaccade task, latency of correct antisaccades, which are controlled by the frontal eye field, ${ }^{14}$ and the percentage of misdirected antisaccades, which are controlled by the prefrontal cortex, ${ }^{13}$ were not significantly increased in patients compared with controls. Therefore, afferent cortical pathways including the lentiform nucleus do not seem to be crucial for the control of antisaccades.

In the group of paradigms requiring internal representation of target properties, patients with lesions of the lentiform nucleus showed significant impairment in performance compared with controls. A significant increase in the percentage of errors was found in patients tested with saccade sequences. Saccade sequences are also disturbed in patients with lesions affecting the supplementary eye field, ${ }^{18}$ which lies in the rostral part of the supplementary motor area ${ }^{19}$ and, at the cortical level, controls such sequences. Moreover, in patients with Parkinson's disease, an impairment of saccade sequences, improved by treatment with levodopa, has been reported. ${ }^{20}$ PET studies have shown that the dysfunction of the supplementary motor area in patients with Parkinson's disease is improved by levodopa. ${ }^{21}$ Accordingly, it may be that the control of saccade sequences by the supplementary eye field depends on a subcortical pathway, probably originating in the substantia nigra pars compacta $(\mathrm{SNpc}$ ) and joining in the putamen the pathway which projects to the frontal lobe (via the pallidum and thalamus, see earlier). Therefore, the involvement of such a pathway in saccade sequences could explain why the patients with lesions in the lentiform nucleus performed these sequences incorrectly. However, the specific role of this nucleus in the control of saccade sequences remains to be determined. In monkeys, electrophysiological studies have shown a neuronal activity preceding sequential limb movements both in the supplementary motor area ${ }^{22}$ and the pallidum. ${ }^{23}$ During sequential arm movements, a phasic neuronal activity in the pallidum, influenced by the animal's preparation for movements, is seen. ${ }^{24}$ It has been suggested that such phasic activity is a signal indicating the end of one movement in order to allow the next movement in the sequence to start. ${ }^{24}$ Thus the absence of such a signal after lesions in the lentiform nucleus could explain a disorganisation in the preparation of the next correct saccade in the sequence.

Memory guided saccades are controlled by the prefrontal cortex, ${ }^{25}$ in which spatial working memory could be organised. ${ }^{26}$ Memorised information concerning the spatial location of the target is probably sent by the prefrontal cortex to the frontal eye field, when the input is visual, or to the supplementary eye field, when the input is vestibular. ${ }^{27}$ These different frontal areas receive afferent pathways from the putamen and pallidum via the thalamus. ${ }^{2}$ Therefore, an increase in the amplitude error of memory guided saccades with vestibular input could be explained by a deafferentation of the prefrontal cortex or the supplementary eye field, due to the lesions in the lentiform 
nucleus. The increase in the amplitude error of memory guided saccades with visual input suggests a deafferentation of the prefrontal cortex or the frontal eye field. Amplitude disorders of memory guided saccades have also been found in patients with Parkinson's disease, ${ }^{28}{ }^{29}$ in whom the same nigrostriato-pallidothalamocortical pathway is impaired.

The preservation of antisaccades and the impairment of memory guided saccades, even though both are controlled by the prefrontal cortex,$^{13}{ }^{25}$ could be explained by a difference in the action of the cortical afferent pathways, depending on the saccade to be performed. Perhaps the putamen and pallidum play an important part in the control of intentional, internally triggered saccades, such as memory guided saccades, because, as suggested by PET results, these structures seem to be involved in other types of internally triggered saccades. ${ }^{30}$ On the other hand, it may well be that the putamen and pallidum are not involved or are less involved in the control of unwanted reflexive visually guided saccades, externally triggered in the antisaccade task.

In normal subjects, the existence of a gap in the predictive saccade paradigm results in a significant increase in predictive saccades, suggesting a facilitating effect of an external cue on these internally triggered saccades. In our patients, prediction was possible, because they were as capable of performing predictive saccades as controls in the predictive task without a gap. Such an ability to predict has also been reported in patients with Parkinson's disease. ${ }^{31} 32$ However, the facilitating effect of the gap on predictive saccades no longer existed in our patients, suggesting that such an effect is under the control of the lentiform nucleus. It should be noted that possibly in relation to this facilitating effect of the gap on predictive saccades, the aforementioned phasic activity of the pallidal neurons ${ }^{24}$ seems to be dependent on the context of the next movement; in particular if this movement is predictable. Thus although the lentiform nucleus does not seem to generate prediction in a saccade task, it could play a part in the modulation of the predictive information used in this task.

In conclusion, our results suggest that the lentiform nucleus controls saccades for which an internal representation of the target properties or a modulation of such information is required. It should be noted that such saccades are also impaired in patients with Parkinson's disease, ${ }^{33}$ in whom a dysfunction of the nigrostriatopallidal pathway also exists. These results confirm the functional role of the basal ganglia circuits, which could influence the cortical areas involved in saccade control as they do for other body movements.

Financial support for this work was provided by the Délégation de la Recherche Clinique de l'A.P.-H.P. (92.2214) and the Faculté de Médecine Pitié-Salpetrière (7581). RM had financial support from the Swiss Parkinson Association.

1 Pierrot-Deseilligny C, Rivaud S, Gaymard B, et al. Cortical control of saccades. Ann Neurol 1995;37:557-67.

2 Alexander GE, Delong MR, Strick PL. Parallel organization of functionally segregated circuits linking basal ganglia and cortex. Ann Rev Neurosci 1986;9:357-81.
3 Yeterian EH, Van Hoesen GW. Cortico-striate projections in the rhesus monkey: the organization of certain corticocaudate connections. Brain Res 1978;139:43-63.

4 Van Hoesen GW, Yeterian EH, Lavizzo-Mourey R. Widespread corticostriate projections from temporal cortex of the rhesus monkey. F Comp Neurol 1981;199: 205-19.

5 Selemon LD, Goldman-Rakic P. Longitudinal topography and interdigitation of corticostriatal projections in the rhesus monkey. $\mathcal{F}$ Neurosci $1985 ; 5: 776-94$.

6 Alexander GE, Crutcher MD. Functional architecture of basal ganglia circuits: neural substrates of parallel processing. TINS 1990;13:266-71.

7 Bhatia KP, Marsden CD. The behavioural and motor consequences of focal lesions of the basal ganglia in man. Brain 1994;117:859-76.

8 Laplane D, Levasseur M, Pillon B, et al. Obsessive-compulsive and other behavioural changes with bilateral basal ganglia lesions. Brain 1989;112:699-725.

9 Grober E, Bushke H. Genuine memory deficit in dementia. Developmental Neuropsychology 1987;1II:13-36.

10 Smit AC, VanGisbergen JAM. Short latency transition in saccade dynamics during square-wave tracking and its significance differentiation of visually-guided and predictive saccades. Exp Brain Res 1989;76:64-74.

11 Lapresle J, Fardeau $M$. The central nervous system and carbon monoxyde poisoning. Anatomical study of brain lesions following intoxication with carbon monoxyde. Prog Brain Res 1967;24:31-74

12 Kobayashi K, Isaki K, Fukutani Y, et al. CT Findings of the interval form of carbon monoxide poisoning compared with neuropathological findings. Eur Neurol 1984;23:34 43.

13 Pierrot-Deseilligny C, Rivaud S, Gaymard B, Agid Y. Cortical control of reflexive visually-guided saccades. Cortical control of reflexin 1991;114:1473-85.

14 Rivaud S, Müri RM, Gaymard B, et al. Eye movement disorders after frontal eye field lesions in humans. Exp Brain orders after frontal eye

15 Leichnetz GR, Spencer RF, Hardy SGP, Astruc J. The prefrontal corticotectal projection in the monkey; an anterograde and retrograde horseradish peroxidase study Neuroscience 1981;6:1023-41

16 Segraves MA, Goldberg ME. Functional properties of corticotectal neurons in the monkey's frontal eye field. $f$ Neurophysiol 1987;58:1387-419.

17 Hikosaka O. Role of basal ganglia in saccades. Rev Neurol 1989;145:580-6.

18 Gaymard B, Rivaud S, Pierrot-Deseilligny C. Role of the left and right supplementary motor areas in memoryleft and right supplementary motor areas in memol 1993;28:622-6.

19 Schlag J, Schlag-Rey M. Evidence for a supplementary eye field. F Neurophysiol 1985;57:179-200.

20 Vermersch Al, Rivaud S, Vidailhet $M$, et al. Sequences of memory-guided saccades in Parkinson's disease. Ann Neurol 199:;35:487-90.

21 Jenkins IH, Fernandez W, Playford ED, et al. Impaired activation of the supplementary motor area in Parkinson' disease is reversed when akinesia is treated with apomorphine. Ann Neurol 1992;32:749-57.

22 Mushiake $H$, Inase $M$, Tanji J. Neuronal activity in the primate premotor, supplementary, and precentral motor cortex during visually guided and internally determined sequential movements. F Neurophysiol 1991;66:705-18.

23 Mushiake H, Strick PL. Activity of pallidal neurons during sequential movements. Society for Neuroscience Abstracts 1993;19:1584

24 Brotchie P, Iansek R, Horne MK. Motor function of the monkey globus pallidus: cognitive aspects of movemen monkey globus pallidus: cognitive aspects of movement

25 Pierrot-Deseilligny $C$, Rivaud S, Gaymard B, Agid Y. Cortical control of memory-guided saccades in man. Exp Brain Res 1991;83:607-17.

26 Goldman-Rakic PS. Circuitry of primate prefrontal cortex and regulation of behavior by representational memory. In: Plum F, Mountcastle VB, eds. Handbook of physiology The nervous system. Vol 5, Bethesda: The American Physiological Society, 1987:373-417.

27 Pierrot-Deseilligny C, Israel I, Berthoz A, et al. Role of the different frontal lobe areas in the control of the horizontal component of memory-guided saccades in man. Exp Brain Res 1993;95:166-71.

28 Crawford TJ, Henderson L, Kennard C. Abnormalities of nonvisually-guided eye movements in Parkinson's disease. Brain 1989;112:1573-86.

29 Lueck CJ, Tanyeri S, Crawford TJ, Henderson L, Kennard C. Antisaccades and remembered saccades in Parkinson's C. Antisaccades and remembered saccades in Parkins.

30 Petit L, Orssaud C, Tzourio N, et al. PET study of voluntary saccadic eye movements in humans: basal gangliatary saccadic eye movements in humans: basal gangliaPhysiol 1993;69:1009-17.

31 Crawford TJ, Gennard C. Predictive responses in Parkinson's disease: manual keypresses and saccadic eye movements to regular stimulus events. F Neurol Neurosurg Psychiatry 1989;52:1033-42.

32 Ventre J, Zee DS, Papageorgious H, Reich S. Abnormalitie of predictive saccades in hemi-Parkinson's disease. Brain 1992;115:1147-65.

33 Kennard C, Crawford TJ, Henderson L. A pathological approach to saccadic eye movements in neurological and psychiatric disease. $\mathcal{F}$ Neurol Neurosurg Psychiatry 1994;57: $881-5$ 